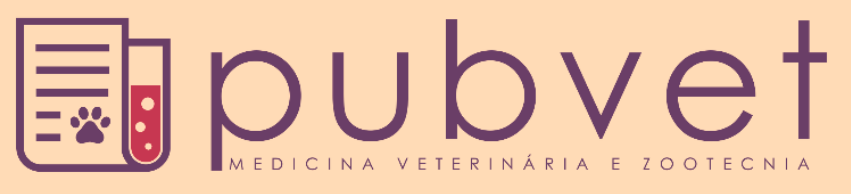

https://doi.org/10.31533/pubvet.v16n02a1046.1-5

\title{
Reação inflamatória ao fio de náilon pós ovariohisterectomia em coelha obesa: Relato de caso
}

\author{
Alexandre Longo Filho ${ }^{1 *}{ }^{\bullet}$, Maritza Nunes Severiano ${ }^{1} \bullet$, Thais Jassek Soares ${ }^{1}{ }^{\bullet}$, Danielle \\ Shiguihara $^{1}{ }^{\ominus}$, Letícia Burigo Inácio ${ }^{\circ}{ }^{\circ}$, Amanda Souto Sarti ${ }^{1}{ }^{\ominus}$ \\ ${ }^{1}$ Médico(a) veterinário(a) autônomo(a). Curitiba-PR Brasil. \\ ${ }^{2}$ Discente de Medicina Veterinária - Universidade Federal do Paraná (UFPR). Curitiba-PR. \\ *Autor para correspondência, E-mail: longofilho1@hotmail.com
}

\begin{abstract}
Resumo. A técnica de ovariohisterectomia é comumente realizada de forma eletiva ou terapêutica na rotina veterinária. O profissional deve estudar a fundo o quadro do paciente, entendendo os fatores que podem interferir no prognóstico. A escolha do fio, o escore corporal e o estado geral do paciente devem ser levados em consideração buscando a adequação da técnica. Uma coelha, Lion Head, de cinco anos e 2,650 kg foi atendida na Clínica Veterinária Palma Pereira e diagnosticada com infecção uterina. Foi realizada cirurgia de ovariohisterectomia usando fio de náilon. Um ano após a cirurgia a paciente retornou apresentando uma massa neoformada intra-abdominal. Realizou-se a exérese da formação e exame histopatológico, aferindo o seu caráter inflamatório. Pode-se concluir que neste caso a infecção uterina, o fio utilizado no procedimento cirúrgico e o excesso de peso foram fatores predisponentes a reação inflamatória exagerada, todavia se aponta a necessidade da realização de maiores estudos sobre o tema.
\end{abstract}

Palavras-chave: Coelha, histopatológico, náilon, ovariohisterectomia

\section{Inflammatory reaction to nylon suture after ovariohisterectomy in obese rabbit: Case report}

\begin{abstract}
The ovariohysterectomy is commonly performed in the veterinary routine, either electively or therapeutically. The professional must thoroughly study the patient's condition, understanding the factors that may interfere with the prognosis. The choice of thread, patient's body score and general condition of the patient must be taken into account in order to adapt the technique. A five-year-old, Lion Head, rabbit weighting 2,650 kg was treated at the Clínica Veterinária Palma Pereira and diagnosed with uterine infection. Ovariohysterectomy surgery with nylon thread was performed. One year after surgery, the patient returned with an intra-abdominal mass. The neoformation was resected histopathological examination stated its inflammatory character. It can be concluded that uterine infection, the thread used in the surgical procedure and overweight were predisposing factors to an exaggerated inflammatory reaction in this case. There is a need for further studies on this topic.
\end{abstract}

Keywords: Rabbit, histopathological, nylon, ovariohysterectomy

\section{Introdução}

A escolha do fio de sutura é de suma importância para o sucesso de um procedimento cirúrgico, sendo que tecidos e técnicas apresentam diferentes necessidades (MacPhail, 2014). Todos os fios de sutura levam à reação por corpo estranho pelo organismo, logo é imprescindível conhecer as características físicas e químicas do material a ser utilizado (Rahal et al., 1998). 
O fio de náilon é constituído a partir de poliamida, isento de capilaridade e monofilamentar (Gianlupi \& Trindade, 2004). O material é considerado permanente, embora ocorra uma lenta liberação de radicais livres bacteriostáticos de poliamida devido a reação de hidrólise (Campbell \& Marks, 1985).

No trabalho realizado por Simeonova \& Danchev (2013) concluiu-se que o fio de poliamida não causa reação intracutânea em coelhos. Em contraponto, o fio de náilon apresentou eventos de proliferação fibroblástica e neoformação vascular precoce, juntamente com a presença de células gigantes de corpo estranho (Ribeiro et al., 2005). Em resposta a corpos estranhos, o sistema imune dos coelhos reage para formar material caseoso e, por conta disso, são mais propensos a reagir adversamente aos materiais de sutura (Harcourt-Brown \& Harcourt-Brown, 2002; Saunders \& Davies, 2005). Essa resposta também envolve o excesso de tecido de granulação, tornando comuns as complicações pósoperatórias por reação à sutura (Harcourt-Brown \& Chitty, 2013).

Coelhos obesos são maus candidatos a procedimentos cirúrgicos (Harcourt-Brown \& HarcourtBrown, 2002; Saunders \& Davies, 2005). Segundo a clínica de coelhos da universidade de Edinburgh, a cada quatro animais um é obeso (Reusch, 2010).

Os coelhos são muito utilizados como modelos para avaliação de reação ao fio no pós-operatório, formação de adesões, bem como para estudos de obesidade em humanos (Harcourt-Brown \& HarcourtBrown, 2002; Saunders \& Davies, 2005). Todavia, são escassas as publicações que mostram a aplicabilidade dessas informações na rotina clínica. Levando isso em consideração, o objetivo deste trabalho foi relatar um caso em que a reação ao fio de náilon e a obesidade interferiram na conduta clínica.

\section{Relato de caso}

Uma coelha, cinco anos de idade, com 2,650 kg (peso ideal 1,8 kg), da raça Lion Head chegou para consulta na Clínica Veterinária Palma Pereira - Florianópolis. A partir do exame clínico, associado aos exames laboratoriais e de imagem, diagnosticou-se infecção uterina, sendo realizada a cirurgia de ovariosalpingohisterectomia $(\mathrm{OSH})$. O fio de náilon foi utilizado para ligaduras e suturas internas. Não houve intercorrências no pós-operatório.

Um ano após a cirurgia a paciente retornou à clínica, devido a presença de piolhos. Todavia, durante o exame físico de rotina notou-se uma massa indefinida com três $\mathrm{cm}$ de diâmetro na cavidade pélvica. Foi requisitado a ultrassonografia e evidenciou-se a presença de uma formação ovalada de $3,0 \mathrm{~cm}$ x 1,8 $\mathrm{cm}$, com cápsula pouco definida, parênquima de aspecto heterogêneo, área cranial com aumento de ecogenicidade e leve formação de sombra acústica, não vascularizado, em região hipogástrica central, cranial a bexiga urinária. As alterações eram sugestivas de neoformação, fibrose ou calcificação.

Os tutores optaram por realizar acompanhamento ultrassonográfico. O exame foi repetido um mês depois e apresentou as mesmas características citadas no primeiro exame; porém, com uma nova formação de menor diâmetro $(1,3 \mathrm{~cm})$. Além disso, ficou mais evidente o acúmulo de tecido adiposo em região hipogástrica.

Um mês após a visualização das estruturas, foi realizado a exérese dos dois focos tumorais em tecido adiposo abdominal. O maior deles estava aderido ao coto uterino e o menor sem ligação direta. Foi utilizado fio sintético absorvível (poliglactina) para ligamento dos vasos e laparorrafia. Foi receitado para o pós-operatório dipirona ( $1 \mathrm{gota} / \mathrm{kg})$ por cinco dias, três vezes ao dia, tramadol $(12 \mathrm{mg} / \mathrm{kg})$ por cinco dias, duas vezes ao dia, meloxicam $(0,4 \mathrm{mg} / \mathrm{kg})$ por dois dias, uma vez ao dia e repouso em lugar restrito por sete dias. A massa foi enviada para exame histopatológico, com suspeita de adenocarcinoma de coto uterino.

No exame histopatológico não foi evidenciado células neoplásicas, tendo como diagnóstico reação inflamatória, cujas características foram definidas por proliferação de tecido de granulação maduro e conjuntivo fibroso, alterações vasculares e infiltração difusa de histiócitos. Evidenciou-se um padrão celular majoritariamente mononuclear, com poucos neutrófilos. A conclusão foi esteatite crônica ativa proliferativa com necrose e mineralização distrófica por corpo estranho.

Uma semana após a cirurgia o animal estava alerta e ativo, apresentando completa cicatrização cutânea. Todavia, na palpação abdominal foi notado uma hérnia abdominal com 4,0 cm x 3,0 cm. Foi 
realizado cirurgia para correção da hérnia. A medicação pré-operatória utilizada foi quetamina (5 $\mathrm{mg} / \mathrm{kg})$, midazolam $(0,5 \mathrm{mg} / \mathrm{kg})$ e butorfanol $(0,2 \mathrm{mg} / \mathrm{kg})$. A indução e manutenção foi feita por anestesia inalatória com isofluorano. Durante o procedimento foi evidenciado que se tratava de uma hérnia incisional, também chamada de hérnia falsa ou eventração. Realizou-se a síntese da musculatura com fio absorvível sintético, com pontos simples separado, seguindo para a síntese da pele com fio de náilon, com pontos em colchoeiro. Não houve intercorrência no pós-operatório.

\section{Discussão}

A desordem uterina mais comum em coelhos é o adenocarcinoma (Künzel et al., 2015), podendo se desenvolver no coto uterino remanescente após OSH (Brown et al., 2010; Naor et al., 2017), sendo este um dos diferenciais deste caso. Além das neoplasias, a piometra é mais comumente encontrada em coelhas destinadas a reprodução e mais rara em animais de criação (Quinton, 2005). A rápida formação de aderências em coelhos dificulta o tratamento destas afecções (Harcourt-Brown \& Harcourt-Brown, 2002; Saunders \& Davies, 2005).

Künzel et al. (2015) demonstraram que em 50\% dos pacientes com desordens uterinas, a palpação abdominal foi útil para o diagnóstico, revelando distensões ou massas irregulares. Todavia, essa preocupação deve ser estendida a animais castrados, pois podem desenvolver infecção do coto uterino após a OSH (Harcourt-Brown \& Harcourt-Brown, 2002; Künzel et al., 2015). As principais complicações pós-cirúrgicas envolvem hemorragia, necrose de gordura, aderência, infecção e anorexia pós-operatória (Harcourt-Brown \& Harcourt-Brown, 2002). O animal deste relato não apresentou complicações no pós-operatório imediato, todavia foram evidenciadas aderência e necrose na exérese da massa e na histopatologia. Os sinais clínicos decorrentes de alterações no coto uterino são inespecíficos, e inclui letargia, desidratação leve, taquipneia, taquicardia e febre (Campbell, 2004). O animal neste caso era assintomático e a alteração foi um achado do exame clínico de rotina.

Os fios utilizados na sutura são os causadores de grande parte das reações de coto uterino (Boza et al., 2010). Outrossim, a infecção residual após a castração em animal acometido por piometra pode levar a esse quadro (Campbell, 2004). Tais fatores estão em consonância com o caso, visto que durante o procedimento se utilizou fio não absorvível em um tecido acometido por piometra, aumentando a chance de infecção ou inflamação residual. Quando utilizado em suturas profundas, o fio de náilon é considerado pelo organismo como um corpo estranho, levando a proliferação de fibroblastos e capilares sanguíneos de forma precoce na região de contato (Ávila Filho et al., 2015; Hering \& Gabor, 1993). No decorrer de uma semana já é possível evidenciar células gigantes, tecido conjuntivo bem desenvolvido (Rahal et al., 1998; Weismann \& Brito, 1993) e fibrose, perpetuando a reação tecidual (Ribeiro et al., 2005). Foi elucidado por Costa Neto et al. (2014) que a reação inicial causada por esse tipo de fio se apresenta de forma aguda, com padrão celular misto. Transcorrido duas semanas, a reação passa a ter características crônico-ativa e por fim, crônico-reparadora, com padrão celular mononuclear.

Uma reação local branda pode ser reversível, mas se houver exacerbação da resposta, excedendo certos limites, ocorre morte celular. Assim sendo, evidencia-se áreas de necrose próximas ao sítio de reparação tecidual, levando a cronificação da reação. Os tecidos acometidos são passíveis de mineralização distrófica, devido ao acúmulo de cálcio circulante nas mitocôndrias e pelo influxo aumentado de cálcio, mesmo com níveis de cálcio sérico inalterados (Zachary et al., 2012).

Na resposta inflamatória crônica, caso o estímulo inicial e subsequente não tenha sido resolvido, se observa uma inflamação crônica ativa (Zachary et al., 2012). O resultado do exame histopatológico destacou a proliferação de tecido conjuntivo fibroso, com maior presença de células mononucleares, em concordância com a literatura. A presença de poucos neutrófilos, alterações vasculares e a necrose de gordura com mineralização caracterizam o quadro proliferativo crônico ativo.

O coelho apresentava $40 \%$ a mais de peso corporal $(2,650 \mathrm{~kg})$ do que o ideal $(1,800 \mathrm{~kg})$. A obesidade é um fator de persistência da inflamação devido a maior liberação de citocinas pró-inflamatórias pelos adipócitos (Teng et al., 2018) e super pressão de COX-2 (Alarcon et al., 2018). A cronicidade da inflamação pode atuar como gatilho para estabelecer o quadro de síndrome metabólica, levando a desordem da função celular e vascular (Tziomalos et al., 2010). É possível inferir, por conseguinte, que a reação exacerbada do paciente deste relato pode ser fundamentada pelo histórico de infecção uterina, 
utilização de material de sutura não absorvível e escore corporal elevado, gerando estímulo inflamatório constante cuja resposta levou a formação de uma massa de gordura com esteatite crônica ativa e proliferativa, com necrose e mineralização distrófica.

\section{Conclusão}

Mais estudos devem ser realizados avaliando a resposta inflamatória em coelhos obesos após cirurgias utilizando fio de náilon. Sugere-se maior número de amostras e grupo controle, além do acompanhamento contínuo por ultrassom dos animais castrados. O Médico Veterinário deve estar ciente das possíveis complicações das situações descritas, assim como dos diagnósticos diferenciais.

\section{Referências bibliográficas}

Alarcon, G., Roco, J., Medina, M., Medina, A., Peral, M., \& Jerez, S. (2018). High fat diet-induced metabolically obese and normal weight rabbit model shows early vascular dysfunction: Mechanisms involved. International Journal of Obesity, 42(9), 1535-1543. https://doi.org/10.1038/s41366-018-0020-6.

Ávila Filho, S. H., Lamaro, L., Queiroz, P. J. B., Ferreira, K. D., \& Silva, L. A. F. (2015). Aspectos gerais dos fios de sutura utilizados ou com potencial aplicabilidade na medicina veterinária. Enciclopédia Biosfera, 11(22), 319-350. https://doi.org/http://dx.doi.org/10.18677/Enciclopedia_Biosfera_2015_088.

Boza, S., Lucas, X., Zarelli, M., Soler, M., Belda, E., \& Agut, A. (2010). Late abscess formation caused by silk suture following hysterectomy in a female dog. Reproduction in Domestic Animals, 45(5), 934-936. https://doi.org/10.1111/j.1439-0531.2009.01366.x.

Brown, Y. K., Knowles, S., Fiorello, C. V, Camus, M. S., \& Howerth, E. W. (2010). Pathology in practice. Journal of the American Veterinary Medical Association, 237(11), 1257-1259. https://doi.org/10.2460/javma.237.11.1257.

Campbell, B. G. (2004). Omentalization of a nonresectable uterine stump abscess in a dog. Journal of the American Veterinary Medical Association, 224(11), 1799-1803. https://doi.org/10.2460/javma.2004.224.1799.

Campbell, J., \& Marks, A. (1985). Suture materials and suturing techniques. In Practice, 7(3), 72-75. https://doi.org/10.1136/inpract.7.3.72.

Costa Neto, J., Lima, A., Oriá, A., Martins Filho, E., \& Teixeira, D. (2014). Análise histopatológica das reações teciduais produzidas pelo implante de fio e de braçadeira de náilon. Enciclopédia Biosfera, 10(18), 291-298.

Gianlupi, Á., \& Trindade, M. R. M. (2004). Comparação entre o uso de fio inabsorvível (polipropileno) e fio absorvível (poliglactina 910) na fixação de prótese de polipropileno em correção de defeitos músculo-aponeurótico da parede abdominal. Estudo experimental em ratos. Acta Cir Bras, 19(2), 94-102.

Harcourt-Brown, F., \& Chitty, J. (2013). BSAVA manual of rabbit surgery, dentistry and imaging. In BSAVA Manual of rabbit surgery, dentistry and imaging. British Small Animal Veterinary Association.

Harcourt-Brown, F., \& Harcourt-Brown, N. H. (2002). Textbook of rabbit medicine. ButterworthHeinemann.

Hering, F. L. O., \& Gabor, S. (1993). Propriedades dos fios de sutura. In H. F.L.O., G. S., \& R. D. (Eds.), Bases técnicas e teóricas de fios e suturas (pp. 9-17). Roca, Brasil.

Künzel, F., Grinninger, P., Shibly, S., Hassan, J., Tichy, A., Berghold, P., \& Fuchs-Baumgartinger, A. (2015). Uterine disorders in 50 pet rabbits. Journal of the American Animal Hospital Association, 5l(1), 8-14. https://doi.org/10.5326/jaaha-ms-5812.

MacPhail, C. M. (2014). Cirurgia dos sistemas reprodutivo e genital. In T. W. Fossum (Ed.), Cirurgia de pequenos animais. Elsevier.

Naor, A. W., Wilkerson, M. J., Meindel, M., Morton, M., \& Pohlman, L. M. (2017). Pathology in practice. Journal of the American Veterinary Medical Association, 250(6), 627-629. https://doi.org/10.2460/javma.250.6.627. 
Quinton, J.-F. (2005). Novos Animais de Estimação-Pequenos Mamíferos. Editora Roca.

Rahal, S. C., Rocha, N. S., Figueiredo, L. A., \& Iamaguti, P. (1998). Estudo comparativo das reações teciduais produzidas pela linha de pesca (poliamida) e fio de náilon cirúrgico. Ciência Rural, 28(1), 89-93.

Reusch, B. (2010). Why do I need to body condition score my rabbit? Rabbiting On, 10-11.

Ribeiro, C. M. B., Silva Júnior, V. A., Silva Neto, J. C., \& Vasconcelos, B. C. E. (2005). Estudo clínico e histopatológico da reação tecidual às suturas interna e externa dos fios monofilamentares de nylon e poliglecaprone $25 \mathrm{em}$ ratos. Acta Cirúrgica Brasileira, 20, 284-291. https://doi.org/10.1590/S0102-86502005000400005

Saunders, R. A., \& Davies, R. R. (2005). Notes on rabbit internal medicine. Black Acaddemic \& Professional.

Simeonova, R., \& Danchev, N. (2013). Assessment of surgical sutures Polymed® by intracutaneous irritation test in rabbits. Interdisciplinary Toxicology, 6(2), 99-102. https://doi.org/10.2478/intox2013-0017.

Teng, K. T., McGreevy, P. D., Toribio, J., Raubenheimer, D., Kendall, K., \& Dhand, N. K. (2018). Associations of body condition score with health conditions related to overweight and obesity in cats. Journal of Small Animal Practice, 59(10), 603-615. https://doi.org/10.1111/jsap.12905.

Tziomalos, K., Athyros, V. G., Karagiannis, A., \& Mikhailidis, D. P. (2010). Endothelial dysfunction in metabolic syndrome: prevalence, pathogenesis and management. Nutrition, Metabolism and Cardiovascular Diseases, 20(2), 140-146. https://doi.org/10.1016/j.numecd.2009.08.006.

Weismann, R., \& Brito, J. H. M. (1993). Resposta tecidual frente a diferentes fios de sutura implantados na língua de ratos. Revista Odonto Ciência, 8(16), 19-31.

Zachary, J. F., McGavin, D., \& McGavin, M. D. (2012). Bases da patologia em veterinária. Elsevier Brasil.

Histórico do artigo:

Recebido: 10 de outubro de 2021

Aprovado: 1 de dezembro de 2021

Disponível online: 28 de fevereiro de 2022
Licenciamento: Este artigoé publicado na modalidade Acesso Aberto sob a licença Creative Commons Atribuição 4.0 (CC-BY 4.0), a qual permite uso irrestrito, distribuição, reprodução em qualquer meio, desde que o autor e a fonte sejam devidamente creditados. 